\title{
DNA replication arrest leads to enhanced homologous recombination and cell death in meristems of rice OsRecQ|4 mutants
}

Yong-lk Kwon ${ }^{1,4,5}$, Kiyomi Abe ${ }^{2}$, Masaki Endo ${ }^{1}$, Keishi Osakabe ${ }^{3}$, Namie Ohtsuki ${ }^{1}$, Ayako Nishizawa-Yokoi ${ }^{1}$, Akemi Tagiri ${ }^{1}$, Hiroaki Saika ${ }^{1}$ and Seiichi Toki ${ }^{1,4,5^{*}}$

\begin{abstract}
Background: Mammalian BLM helicase is involved in DNA replication, DNA repair and homologous recombination (HR). These DNA transactions are associated tightly with cell division and are important for maintaining genome stability. However, unlike in mammals, cell division in higher plants is restricted mainly to the meristem, thus genome maintenance at the meristem is critical. The counterpart of BLM in Arabidopsis (AtRecQ4A) has been identified and its role in HR and in the response to DNA damage has been confirmed. However, the function of AtRecQ4A in the meristem during replication stress has not yet been well elucidated.

Results: We isolated the BLM counterpart gene OsRecQ/4 from rice and analyzed its function using a reverse genetics approach. Osreca/4 mutant plants showed hypersensitivity to DNA damaging agents and enhanced frequency of HR compared to wild-type (WT) plants. We further analyzed the effect of aphidicolin —an inhibitor of S-phase progression via its inhibitory effect on DNA polymerases-on genome stability in the root meristem in osrecq/4 mutant plants and corresponding WT plants. The following effects were observed upon aphidicolin treatment: a) comet assay showed induction of DNA double-strand breaks (DSBs) in mutant plants, b) TUNEL assay showed enhanced DNA breaks at the root meristem in mutant plants, c) a recombination reporter showed enhanced HR frequency in mutant calli, d) propidium iodide (PI) staining of root tips revealed an increased incidence of cell death in the meristem of mutant plants.
\end{abstract}

Conclusions: These results demonstrate that the aphidicolin-sensitive phenotype of osrecq/4 mutants was in part due to induced DSBs and cell death, and that OsRecQI4 plays an important role as a caretaker, maintaining genome stability during DNA replication stress in the rice meristem.

Keywords: Rice, RecQ helicase, OsRecQ|4, DNA replication, DNA double-strand break, Homologous recombination, Meristem

\section{Background}

DNA damage is thought to be induced not only by exogenous genotoxic stresses such as UV and ionizing radiation but also by intrinsic endogeneous stresses such as DNA replication errors and the oxidative stress associated with cell metabolism. Unlike animals, plants cannot escape from exogenous genotoxic stresses.

\footnotetext{
* Correspondence: stoki@affrc.go.jp

'Plant Genome Engineering Research Unit, National Institute of

Agrobiological Sciences, 2-1-2 Kannondai, Tsukuba, Ibaraki 305-8602, Japan ${ }^{4}$ Graduate School of Nanobioscience, Yokohama City University, 22-2, Seto, Kanazawa, Yokohama 236-0027, Japan

Full list of author information is available at the end of the article
}

Furthermore, in plants, DNA replication sites are restricted mainly to meristematic tissues, and differentiation of the germ line is not predetermined; plants thus need an efficient and flexible genome maintenance system. However, very little is known about the mechanism maintaining genome stability in response to DNA replication stresses.

RecQ helicases are members of the ATP-dependent helicase family and play important roles in maintaining genome stability in eukaryotic cells during DNA repair [1,2], DNA replication [3,4], telomere maintenance [5], and homologous recombination (HR) processes [6,7].

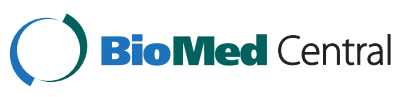


Yeast has a single RecQ gene, Sgs1, but mammals and plants have multiple RecQ genes, humans having five and Arabidopsis seven [8]. Interestingly, overlapping and distinct roles of RecQ proteins have been reported in humans [6,9]. For example, defects in BLM, WRN and HsRecQ4 are found in Bloom's, Werner, and RothmundThomson syndromes, respectively [10-12]. Patients with these syndromes exhibit genomic instability and are predisposed to cancer, and their cells are hypersensitive to DNA-damaging agents. During DNA replication and maintenance of the telomere, the role of BLM overlaps with that of WRN. However, BLM promotes exonuclease 1-mediated DNA resection during the initial step of DSB repair, whereas other RecQ helicases do not promote this step [13,14]. Strikingly, patients with the $B L M$ defect show enhanced sister chromatid exchange (SCE) frequencies [15], indicating that BLM suppresses HR in the course of DNA replication. Moreover, BLM interacts with replication protein A (RPA) and topoisomerase III $[16,17]$. It has been revealed recently that BLM plays a role in recovery from DNA replication arrest $[18,19]$. Therefore, BLM is likely to be a multi-functional protein involved in several aspects of genome maintenance in mammals. Many studies to date have focused on DNA replication maintenance, given the known association between HR and DNA replication [20-22].

Two BLM homologs, AtRecQ4A and AtRecQ4B, have been isolated in Arabidopsis and their functions analyzed using mutant plants [23,24]. atrecq4A mutants showed enhanced frequency of HR during normal growth condition and hypersensitivity to DNA damaging agents compared to wild-type (WT) plants. In contrast, atrecq4B mutants had a reduced frequency of $\mathrm{HR}$ and did not show sensitivity to DNA-damaging agents compared to WT plants despite the high degree of identity of the two RecQ4s [24]. Recently, it was reported that AtRecQ4A is required for efficient synthesis-dependent strand annealing (SDSA) but has only a limited role in singlestrand annealing (SSA) [25]. Our previous study of OsRecQ14 showed that over-expression of OsRecQl4 promoted the resection process of HR-mediated DSB repair in rice [26]. However, the functions of BLM-related proteins in plants have not been well analyzed, particularly in the context of their potential "caretaker" role during DNA replication in plant meristems.

The presence of seven RecQ-like genes has been reported in rice-a monocotyledonous model plant. However, these seven genes in rice do not correspond to the seven Arabidopsis genes. Interestingly, a single BLM counterpart gene, OsRecQl4, has been predicted in the rice genome $[8,27]$. Although the presence of two BLMlike genes with different functions in Arabidopsis is interesting from the perspective of the evolution of genome stability in plants, the presence of a single $B L M$ counterpart gene in rice seems to favor the functional analysis of BLM counterpart genes. Several previous reports in Arabidopsis had shown the effect of BLM ortholog genes on HR; we asked whether this function could have some relationship to the maintenance of genome stability during DNA replication.

In addition to having an advantage for the analysis of $B L M$ counterpart genes in plants as mentioned above, rice is an ideal model plant in which to evaluate the functions of genes involved in genome stability at sites of DNA replication, i.e., the meristem. Furthermore, S-phase is concentrated in the region of rice root meristems [28]. This is because, in rice, the genotoxic stresses that accompany the arrest of DNA replication never induce endoreduplication [29], which is one of the routes of escape from DNA damage used in Arabidopsis [30].

Here, we report the cloning of OsRecQl4 from rice and characterize the phenotypes of knockout plants in terms of DNA stability at the site of DNA replication. Our findings indicate that OsRecQl4 plays an important role in maintaining genome stability, at least in the root apical meristem (RAM), via suppression of HR.

\section{Results}

\section{Isolation of OsRecQI4 cDNA from rice}

The presence of an AtRecQ4A like sequence in the rice genome was reported by Hartung and Puchta [8], who identified the 1164-amino acids protein CAE03209 (GenBank ID) as OsRecQ4A. We cloned the corresponding full-length CDNA by RT-PCR and RACE using specific primers designed according to the genomic sequence Os04g0433800 containing the CAE3209 coding sequence. Our cDNA sequence encodes 1174 amino acids and is the same as the Loc_Os04g35420.1 sequence predicted in the GRAMENE database [http:// www.gramene.org/]. We named our sequence OsRecQ14 for Oryza sativa RecQ like protein 4.

OsRecQl4 consists of 25 exons and 24 introns, and encodes a protein with 1174 amino acids including the DEXDc (DEAD-like helicase domain including ATP$\mathrm{Mg}^{++}$binding site) and HELICs (helicase superfamily c-terminal including ATP, nucleotide binding site) helicase domains, as well as an RQC (RecQ C-terminal) and an HRDC (required for dissolution of double Holliday junctions) domain [6] (Figure 1A). The result is the same as the prediction by the Rice Genome Annotation Project [http://rice.plantbiology.msu.edu/cgibin/ORF_infopage.cgi?orf=LOC_Os04g35420.1]. Because the DEXDc domain is conserved in BLM and AtRecQ4A, the OsRecQ4A (CAE3209) predicted by Hartung and Puchta lacking the DEXDc domain seems to be nonfunctional (Additional file 1: Figures S1 and S2).

Arabidopsis AtRecQ4A and AtRecQ4B are reported to be paralogous proteins with different functions. Since 


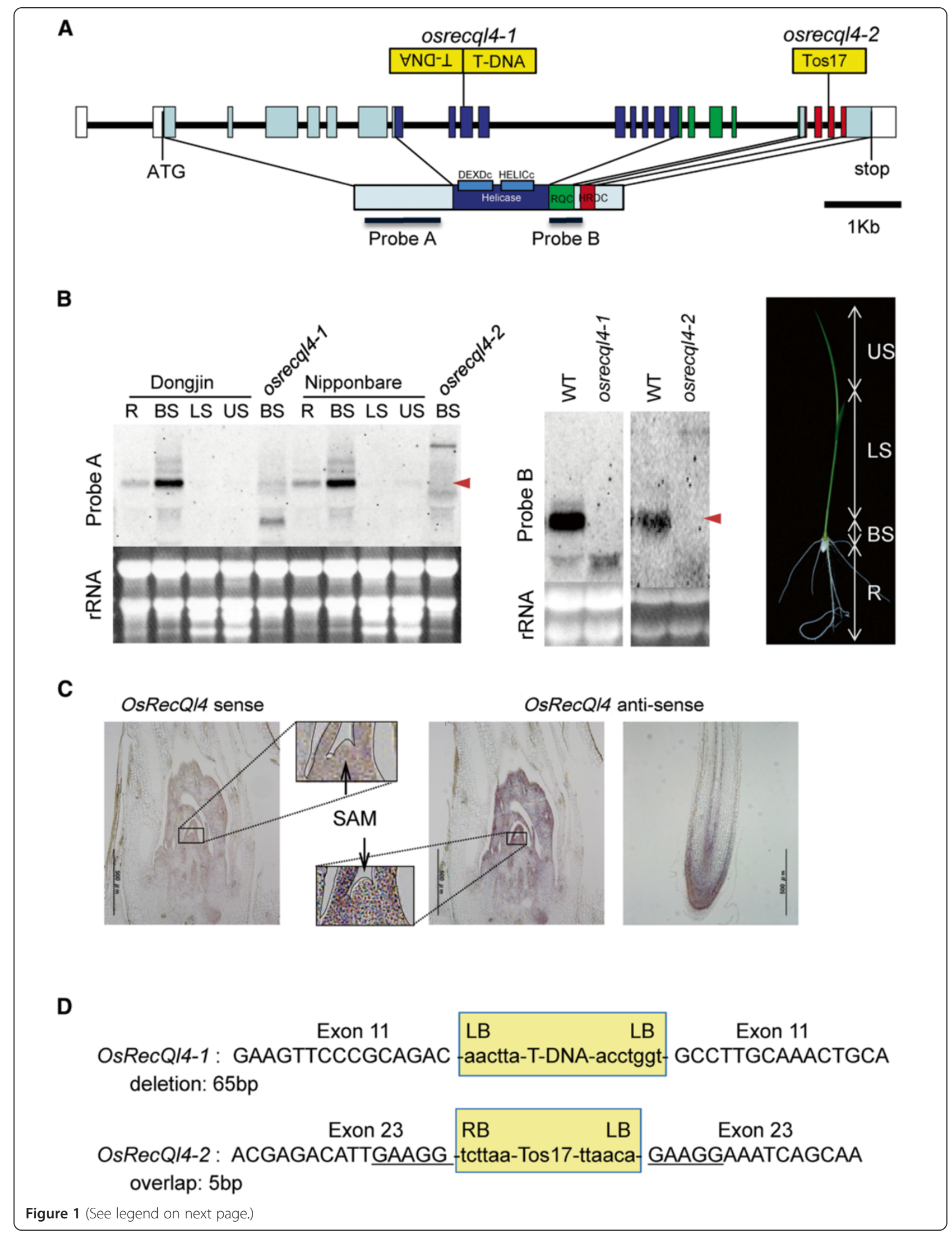


(See figure on previous page.)

Figure 1 Characterization of OsRecQ14 and molecular analysis of knockout lines. (A) T-DNA (from POSTECH) insertion site in OSrecq/4-1, and Tos17 (from NIAS) insertion site in osrecq/4-2. OsRecQ/4 has conserved helicase domains (including DEXDC, and HELICc domain), RecQ C-terminus (RQC), and HRDC domain. Probe A (near the N-terminus, 994 bp), and probe B (part of the RQC domain, 433 bp) were used for northern blot analysis. The T-DNA line shown above the construct harbored a head-to-head insertion in exon 11 . The Tos 17 line carries an insertion in exon 23. (B) Northern blot analysis. OsRecQ/4 mRNA is expressed strongly in the base of shoots (BS) and weakly in roots of both Dongjin and Nipponbare. mRNA was detected by both probes A and B in wild-type (WT) plants but not in osrecq/4-1 or osreca/4-2. Red arrowheads indicate mRNA of OsRecQ/4. The genetic background of T-DNA (osrecq/4-1) is Dongjin, and that of Tos17 (osrecq/4-2) is Nipponbare. 7-day-old seedlings of wild-type (segregated wild type) and mutant (homozygous). R, root; BS, base of shoot; LS, lower shoot; US, upper shoot. (C) In situ hybridization analysis. Anti-sense probes for OsRecQ14 gave strong hybridization signals around the shoot apical meristem (SAM) and the root apical merstem (RAM). OsRecQ|4 is expressed mainly in the meristem. (D) Sequence of the osrecq/4-1 and osrecq/4-2 insertion site. osrecq/4-1 has a genomic deletion of $65 \mathrm{bp}$ in the insertion region, exon 11. osrecal4-2 has a genomic overlap of $5 \mathrm{bp}$ in the insertion region, exon 23.

the amino acids sequences of AtRecQ4A and OsRecQ14 are quite similar to each other, and differ slightly from that of AtRecQ4B protein, we hypothesized that AtRecQ4B protein has acquired or lost specific motifs required for different functions. We compared several motifs of OsRecQ14, AtRecQ4A and AtRecQ4B proteins using the SALAD database [http://salad.dna.affrc.go.jp/ salad/en/] and found that, near the C-terminus of the helicase domain, a CMKMGYNC sequence was detected only in AtRecQ4B; and in the N-terminus of the helicase domain, a DKESQKSQFLSSTATRI sequence is conserved between OsRecQl4 and AtRecQ4A but not in AtRecQ4B (data not shown). Further investigations of these motifs are required. It might be also interesting to try to express either AtRecQ4A or AtRecQ4B protein in the osrecql4 mutant background.

Phylogenetic analysis revealed the isolated OsRecQ14 to be most similar to AtRecQ4A (51.5\%) and AtRecQ4B (51.0\%) at the amino acid level (Additional file 1: Figure S3A). The amino acid sequence encoded by the region spanning exon 8 to exon 16 of OsRecQ14 had especially high identity to that encoded by the region spanning exon 9 to exon 17 of AtRecQ4A (data not shown). As expected, OsRecQ14 more closely resembled AtRecQ4A than AtRecQ4B as determined by BLASTZ alignment analysis [31] (Additional file 1: Figure S3B).

\section{Expression of OsRecQ/4 in meristems}

Expression of OsRecQl4 was analyzed by northern blot in 7-day-old seedlings of the japonica-type rice cultivars Dongjin or Nipponbare. Using probe A (Figure 1A), relatively strong signals were observed in the basal parts of shoots (BS, as defined in Figure 1B) including the shoot apical meristem (SAM), and weak signals were observed in the root (R). No signal was detected in the lower (LS) or upper (US) shoot (Figure 1B).

In addition, SAM- and RAM-specific expression of OsRecQl4 was confirmed by in situ hybridization (Figure 1C). Interestingly, meristem-specific expression of other RecQ family genes in rice was reported by Saotome et al. [27], who analyzed four other RecQ membersOsRecQ1, OsRecQ2, OsRecQsim and OsRecQ886-in rice.
Our result was further supported by a database analysis of RiceXpro [http://ricexpro.dna.affrc.go.jp/] - a repository of gene expression profiles derived from microarray analysis of tissues or organs encompassing all growth stages of rice plants [32]. OsRecQl4 was highly expressed in the RAM (Additional file 1: Figure S4). These results suggested that expression of OsRecQl4 was restricted to meristematic tissues.

To elucidate the biological role of OsRecQl4, we characterized T-DNA and Tos17 retrotransposon inserted lines of OsRecQ14, namely osrecql4-1 (3A-03503 from POSTECH) of Dongjin background and osrecql4-2 (NC2763 from NIAS) of Nipponbare background, respectively. The sequences of the insertion sites were determined by PCR and subsequent sequencing. Two T-DNAs linked in a head-to-head manner were inserted into exon 11 with a genomic deletion of $65 \mathrm{bp}$. The Tos17 line was inserted at exon 23 accompanied by 5 bp overlap (Figure 1A and D).

Expression of OsRecQ14 in osrecql4-1 and osrecql4-2 mutants was evaluated by northern blot analysis. In this experiment, total RNAs from BS in 7-day-old WT and osrecql4 mutants were analyzed. Probe A was located near the $\mathrm{N}$-terminus and probe $\mathrm{B}$ was located near the C-terminus and included part of the RQC domain (Figure 1A). A transcript of ca. $3.5 \mathrm{~kb}$ was detected by probe A and B in WT plants, but not in osrecql4-1 or osrecql4-2 (Figure 1B). Under normal conditions, growth of osrecql4-1 and osrecql4-2 plants was comparable to that of WT plants. Furthermore, these mutant plants were fertile, as also reported for Arabidopsis atrecq4A mutants [23].

\section{OsRecQ14 is involved in DSB repair and recovery from S-phase arrest}

To assess sensitivity to DNA damage, seeds from osrecql4 mutants and their WT counterparts were treated with bleomycin at concentrations between 0 and $20 \mathrm{mg} / \mathrm{L}$. Five days after germination, total root length was measured. When osrecql4-1 or osrecql4-2 mutants were treated with $5 \mathrm{mg} / \mathrm{L}$ bleomycin, root length was shorter than that of WT plants, suggesting that OsRecQl4 might be involved in the repair of DNA single-strand breaks (SSBs) and/or DSBs (Figure 2A). Similarly, we assessed the involvement 


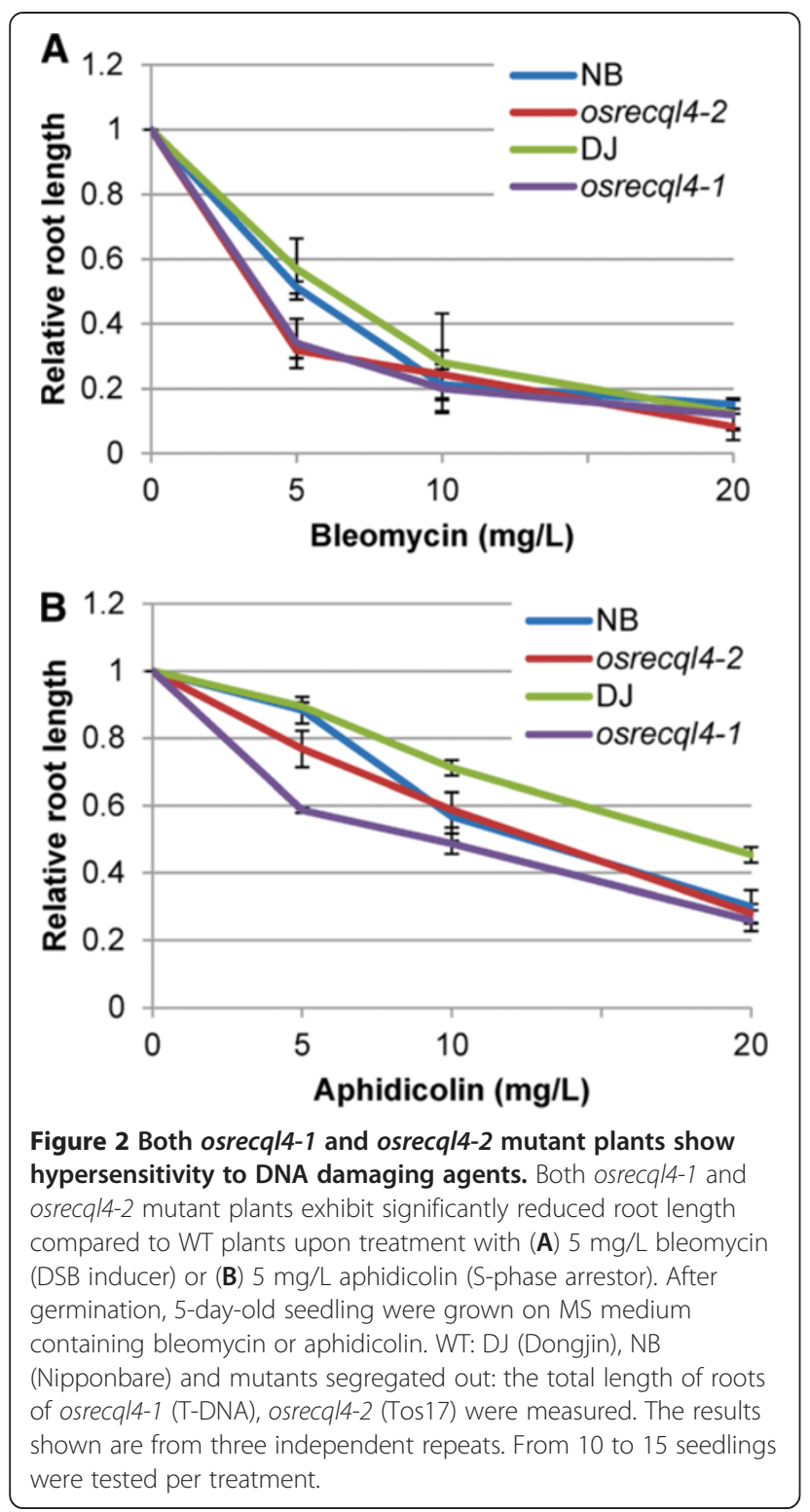

of OsRecQl4 in the recovery process from S-phase arrest caused by inhibition of DNA polymerase. DNA replication can be arrested by aphidicolin-an inhibitor of DNA polymerase $\alpha$ [33]. Decreased root growth was observed upon treatment with $5 \mathrm{mg} / \mathrm{L}$ aphidicolin (Figure 2B). A similar result was obtained upon treatment with $2.5 \mathrm{mM}$ hydroxyurea (data not shown). The expression of OsRecQl4 in roots increased in response to aphidicolin but not bleomycin (Additional file 1: Figure S5).

Judging from the result of northern blot analysis, both osrecql4-1 (T-DNA line) and osrecql4-2 (Tos17 line) mutants have non-functional protein if expressed since OsRecQl4 transcripts were not detected with probe B. Furthermore, both mutants showed hyper-sensitivity against bleomycin and aphidicolin (Figure 2). In addition, we detected increased cell death upon aphidicolin treatment not only in osrecql4-2 (Figure 3) but also in osrecql4-1 (Additional file 1: Figure S7) in our previous manuscript.

Since osrecql4-1 mutant has a GUS expression cassette in its T-DNA region, we could not evaluate homologous recombination frequency using the GU-US recombination substrate system. Therefore, we used mainly the osrecql4-2 mutant line for our experiments.

\section{Failure to recover from DNA replication arrest induces DSBs}

In humans, BLM is involved in the recovery process from DNA replication arrest that can be induced by SSBs or DNA adducts by the unwinding of arrested replication forks. In addition, the increased SCE observed in blm mutant cells is explained by enhanced DSBs due to a lack of the helicase activity of BLM [4]. We therefore investigated whether the increased sensitivity of osrecql4 mutants to aphidicolin treatment was due to a defect of the recovery from DNA replication arrest in osrecql4 cells as like blm mutant cells and a detected increased DSBs in osrecql4 plants.

We used a comet assay to investigate induction of DSBs following aphidicolin treatment. Six-week-old scutellum-derived calli from Nipponbare (NB) and osrecql4-2 were treated with $5 \mathrm{mg} / \mathrm{L}$ aphidicolin for $60 \mathrm{~min}$; the cells were then observed over time after removal of the aphidicolin. The comet tail of osrecql4-2 did not differ from that of control NB without aphidicolin treatment. Following aphidicolin treatment, comet tails indicating DSBs were seen in osrecql4-2 cells immediately after aphidicolin removal, and remained at $15 \mathrm{~min}$ after removal of aphidicolin. In contrast, no comet tails were detected in control NB cells, even after aphidicolin treatment (Figure 4). These results suggest that, in the absence of OsRecQ14, aphidicolin-triggered DNA replication arrest resulted in DSB induction. Although these DSBs might be finally repaired by intrinsic DNA repair systems including $\mathrm{HR}$, cell cycle progression will be delayed by the time needed for DSB repair.

Next, we used TUNEL (terminal deoxynucleotidyl transferase dUTP nick-end labeling) to observe the occurrence of DNA damage in root tips of the osrecql4-2 mutant after aphidicolin treatment. This assay detects DSBs, SSBs, and nicks in tissues by labeling the 3-terminal ends of nucleic acids $[34,35]$. A DNA damage signal was observed in the meristem and in epidermal cells (Figure 5). The former signal is related to cell division but the latter seems to be induced by physical stress during root growth. TUNEL signals induced by physical stress have been observed in root caps and root hairs [36]. DNA damage signals were observed in the root meristem of osrecql4-2 plants but not in WT plants after treatment with $40 \mathrm{mg} / \mathrm{L}$ aphidicolin 

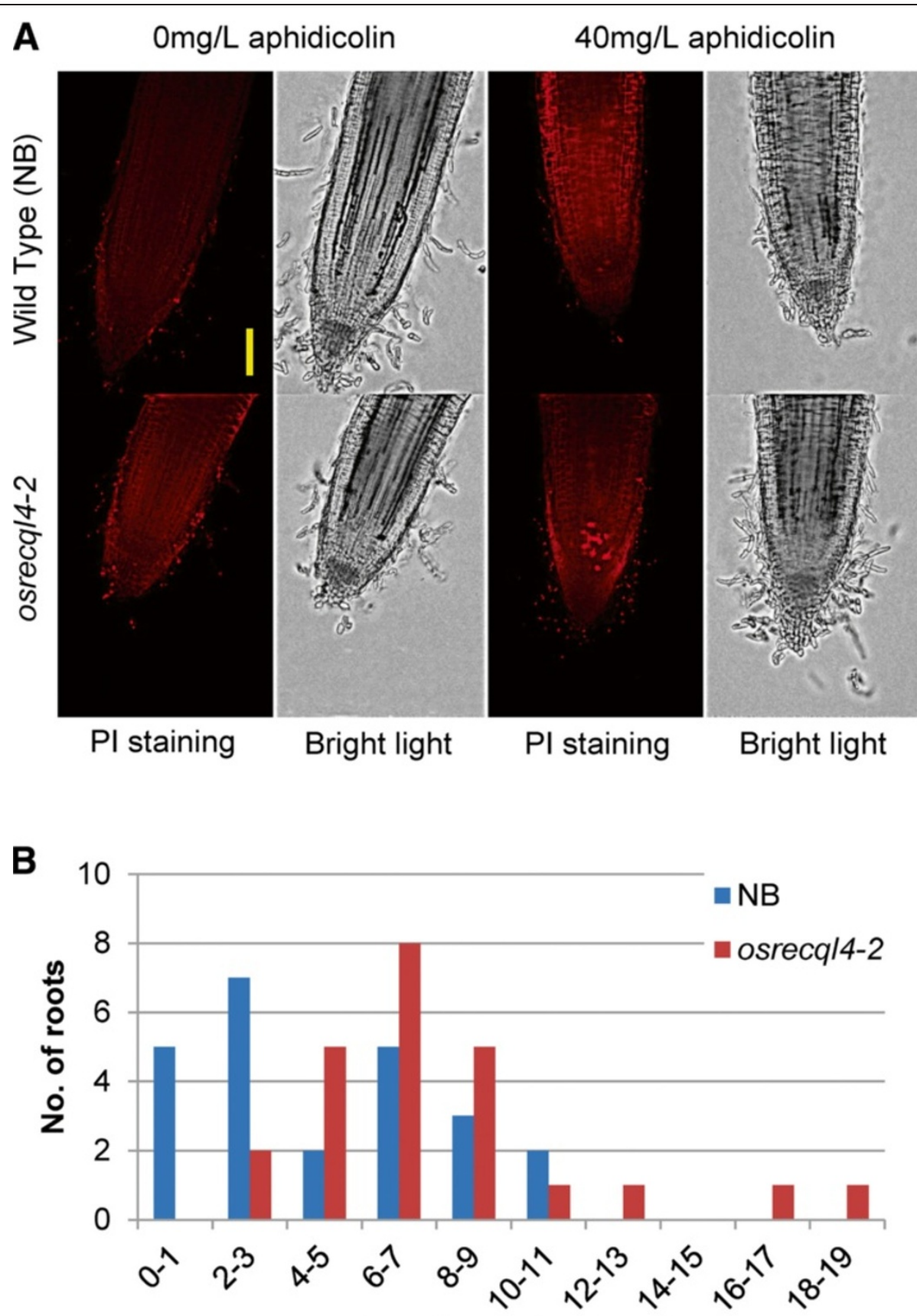

No. of cell death spots

\begin{tabular}{ccc}
\hline & Average of cell death spots & SD \\
\hline NB & 4.46 & \pm 3.35 \\
osrecql4-2 & 7.71 & \pm 3.58 \\
\hline
\end{tabular}


(See figure on previous page.)

Figure 3 Increased frequency of cell death induced in root tips of osrecql4 mutants upon aphidicolin treatment. Propidium iodide (PI) staining indicates dead cells. (A) WT and osrecql4-2 mutant plants treated with $40 \mathrm{mg} / \mathrm{L}$ aphidicolin for $24 \mathrm{~h}$. An increased number of cell death signals was observed in the root meristem of osrecql4-2 under the fluorescence microscope. Scale bar, $100 \mu \mathrm{m}$. (B) Summary of number of PI signals. 24 independent roots from osrecq/4-2 and WT plants were investigated. The x-axis indicates number of cell death spots per root. SD, standard deviation.

(Figure 5). These results indicate that enhanced DNA replication arrest induced DNA damage in the RAM of the osrecql4-2 mutant.

\section{Accumulated DSBs triggered by DNA replication arrest induce $\mathrm{HR}$}

Induced DSBs can be repaired by intrinsic DNA repair systems; if they are not repaired properly, cell death might be induced. Because HR is a DSB repair system used during S-phase of the cell cycle, when sister chromatids are available as templates for DNA repair, we analyzed whether the induced DSBs observed in osrecql4 mutants could enhance HR.

We used the GUS recombination reporter pGU.C.US [37] to monitor the osrecql4 mutant for frequency of HR (Figure 6A). Recombination events between two overlapping GUS sequences can produce a functional GUS gene resulting in blue spots in the cells that can be detected by GUS histochemical staining with 5-bromo-4-chloro3-indolyl glucuronide. pGU.C.US was transformed into a heterozygous osrecql4-2 line; a transgenic line with a single copy of the recombination reporter was identified by Southern blot analysis (Additional file 1: Figure S6) and used for further studies. The frequency of HR events was monitored in the T1 generation of pGU.C.US transgenic rice plants, which were homozygous for either the osrecql4 mutation allele or the WT OsRecQl4 allele. We evaluated the frequency of HR events in scutellum-derived calli.

The distribution of HR frequency by callus weight indicated that HR frequency was greater in the osrecql42 mutant than in WT (Figure 6B). HR frequency did not change in WT but increased further in the osrecql4-2 mutant upon aphidicolin treatment (Figure 6C).

\section{Un-repaired DNA replication arrest induces cell death}

The increased sensitivity to aphidicolin observed in osrecql4 mutants implied that the increased number of DSBs detected in these mutants could induce delayed cell cycle progression or that DSBs might not have been repaired sufficiently by HR.

We revealed cell death by the uptake of PI, which permeates the cell membranes of dying and dead cells [38]. PI-stained root tips were detected after aphidicolin (40 mg/L) treatment for $24 \mathrm{~h}$. We observed root tips using the Z-stack function (see Microscopic analysis in the Methods section). Upon aphidicolin treatment, we detected a significantly increased number of PI staining signals in osrecql4-2 mutants $(7.71 \pm 3.58)$ than in WT NB (4.46 $\pm 3.35 ; \mathrm{P}<0.01$, Mann-Whitney $U$ test) (Figure 3). Similarly, we observed increased PI staining signals in osrecql4-1 mutants $(1.47 \pm 0.18)$ than WT Dongjin $(0.20 \pm 0.14)$ (Additional file 1: Figure S7). The optimum concentration of aphidicolin for the visualization of dead PI-stained cells differed with ecotype. We also used PI staining to see if other types of inducers of DNA damage affected induction of cell death; however, no other inducers led to significant differences between WT and osrecql4 (Additional file 1: Figure S8A). Nevertheless, high concentrations or prolonged treatment induced abnormal shapes in the roots and turned them opaque (Additional file 1: Figure S8B). This result suggested that osrecql4 mutant plants failed to recover from DNA replication arrest, resulting in an increased numbers of DSBs, leading to induction of cell death in the root meristem.

\section{Discussion}

Here, we assayed the function of the BLM homolog OsRecQ14 during DNA replication in rice via knockout mutants. Using the comet assay, we demonstrated induction of DSBs in aphidicolin-treated osrecql4 mutant cells. TUNEL analysis suggested that DNA damage including DSBs was induced at the RAM. The HR assay using the GUS recombination reporter showed that at least a fraction of the DSBs could be repaired by HR, explaining why $\mathrm{HR}$ is enhanced in osrecql4 mutants. The PI staining assay showed that un-repaired DSBs induced cell death at the root meristem. The combined results of comet, TUNEL and HR assays as well as PI staining using mutant plants under a combination of aphidicolin treatments clearly demonstrated the important role of OsRecQl4 in the process of recovery from DNA replication arrest, and also the conserved role of BLM orthologous proteins in this process. In this study, we focused on the role of OsRecQ14 in genomic maintenance during DNA replication in the RAM. However, OsRecQ14 is also expressed in the SAM (Figure 1B, C). Thus, we consider OsRecQl4 to be involved in the maintenance of genome stability during DNA replication at the SAM as well as the RAM. Therefore, increased mutations could accumulate in osrecql4 mutant plants during the mitotic cell cycle, and these mutations should be inherited by subsequent generations.

The osrecql4-1 (T-DNA line) has only a small amount of truncated protein and osrecql4-2 (Tos17 line) has a longer protein according to northern blot analysis 




B
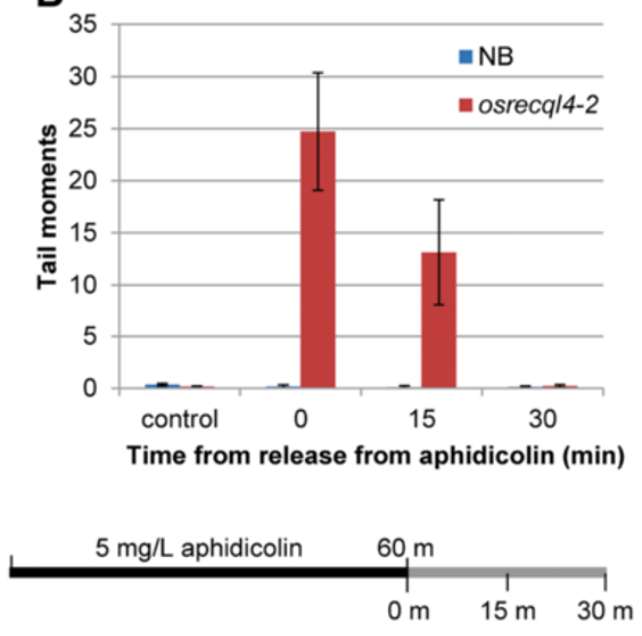

Time from release from aphidicolin

Figure 4 DSBs are induced in osrecql4-2 mutants upon aphidicolin treatment. The comet assay, which can detect DNA breaks at the single cell level, detected DSBs under a neutral conditions protocol. Without aphidicolin treatment, no broken DNA fragments were observed in either wild-type or osrecq/4 cells. Upon treatment with $5 \mathrm{mg} / \mathrm{L}$ aphidicolin for $60 \mathrm{~min}$, broken DNA fragments were observed in osrecq/4-2 cells under the fluorescent microscope (A) as summarized in (B) for osreca/4-2. The DSBs almost disappeared in osreca/4-2 after transferring to aphidicolin-free MS medium for 30 min. Immediately after treatment of aphidicolin; 15 aphidicolin-free MS medium for 15 min after treatment with aphidicolin; 30 aphidicolin-free MS medium for 30 min after treatment of aphidicolin.

(Figure 1B). Thus, we concluded that both the osrecql4-1 and the osrecql4-2 mutants produce an aberrant size of OsRecQ14 protein defective in the consensus domain RQC if expressed. The two mutants might have different effects. However, we revealed both mutants have hypersensitivity to DNA damage agents and increase cell death upon aphidicolin treatment.

In this study, we have shown that DNA replication arrest leads to a hyper-recombination phenotype in plants. Urawa et al. [39] reported that the non-transcribed spacer (NTS) between ribosomal RNA genes (rDNA), which contains a replication fork barrier of rDNA [40], enhances HR in Arabidopsis. In Escherichia coli, it has been hypothesized that a damaged DNA replication fork might be restarted by Holliday junction formation, leading to DNA cleavage by Holliday junction dissolution and finally to repair by HR. However, this mechanism runs the risk of inappropriate recombination. Thus, a stalled fork (not leading to DNA cleavage) might be processed by DNA helicases to avoid replication errors [41].

Recently, Schuermann et al. [42] reported that a defect in DNA polymerase delta $1(P O L \delta 1)$ in Arabidopsis exhibited elevated HR frequency at stalled and collapsed replication forks. This report also supported our conclusion that DNA replication stress induces HR in plants. However, the molecular mechanism connecting DNA replication stress and enhanced HR remains obscure in plants. Here, we see a relationship between accumulated
DSBs, HR and cell death accompanying DNA replication arrest at the site of the meristem.

Rad51-dependent repair HR includes break-induced replication (BIR), double-Holliday junction $(\mathrm{dHJ})$, and SDSA. Rad51-independent SSA also repairs DSB [43]. It was been reported recently that, with a direct repeat GU-US recombination reporter, a functional GUS gene can be generated mainly by the SSA pathway, with SDSA playing only a minor role following DSB [44]. However, during replication, induced DSBs do not induce two free ends, but rather a one-ended DSB, i.e., a DNA double-strand end (DSE). The DSE invades its sister chromatid to be repaired, with DNA synthesis by BIR $[45,46]$. Thus, although increased HR efficiency was demonstrated here using the direct repeat GU-US recombination reporter to associate OsRecQl4 and HR, the HR detected should be Rad51-dependent HR repair. In future, this could be confirmed using a double mutant with Rad51. Further points in support of the hypothesis of alternative repair of the GU-US reporter are the differences in tissues (plant vs. calli) and induction (I-SceI vs. aphidicolin), which might lead to different prevalence of cell-cycle states in which the repair takes place.

We analyzed the sensitivity of osrecql4 mutants to aphidicolin and bleomycin. Since defects in OsRecQ14 enhance the sensitivity of rice to both these latter compounds, OsRecQl4 might be involved in recovery from DNA replication arrest and DSB repair. On the other 


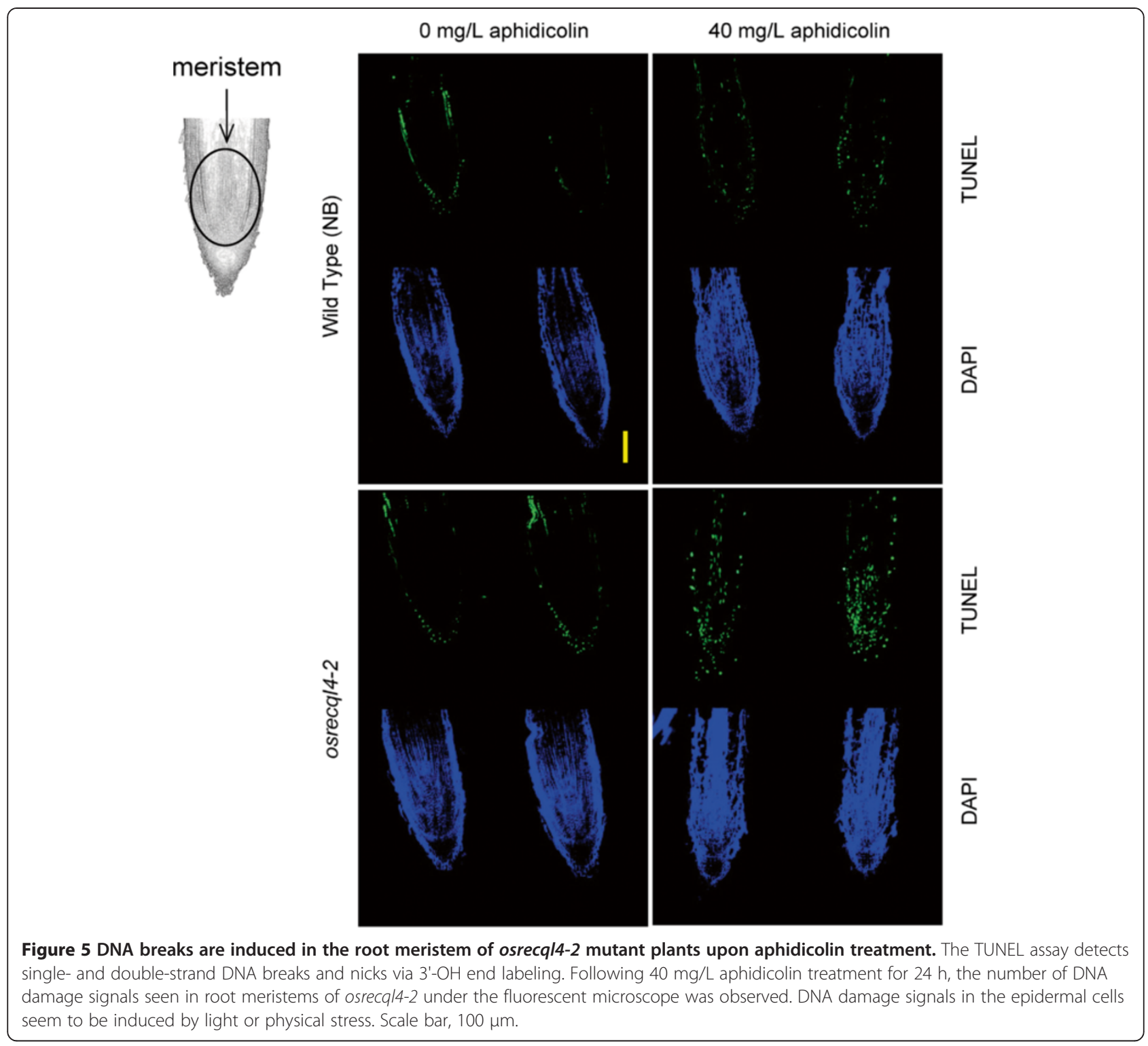

hand, osrecql4 mutants showed normal growth and were fertile, although osrecql4 mutants showed aberrantly sized OsRecQ14 protein, indicating that OsRecQ14 might not be essential. In this respect, it has been reported that members of RecQ family genes in rice-OsRecQ1, OsRecQ2, OsRecQsim and OsRecQ886-are expressed in meristems [27], suggesting that RecQ helicase family members might play overlapping roles in maintaining genome stability in proliferative cells. However, homozygous blm mice exhibit growth retardation [47]. It might be interesting to evaluate growth of the osrecql4 mutant under conditions of elevated UV, since DNA replication arrest is induced by UV photoproducts.

Although the sensitivity of Arabidopsis atrecq4A mutants to bleomycin treatment was the same as that of WT plants, in our study we observed enhanced bleomycin sensitivity in rice osrecql4 mutants compared to WT plants. This might be due to differences in the system, tissue and experimental procedure. Furthermore, we observed cell death in the root meristem of osrecql4 mutant plants. These differences might be attributed to the occurrence of endoreduplication in Arabidopsis, since cells undergoing DNA damage can enter into endocycle and be separated from the mitotic cell cycle in Arabidopsis.

Our results indicate that OsRecQ14 is expressed at SAM and RAM, i.e., sites of cell division. Furthermore, expression of OsRecQ14 was induced by aphidicolin treatment but not by bleomycin (Additional file 1: Figure S5). These results suggest that, according to their level of transcription, OsRecQ14 plays a more important role in repair during DNA replication than in DSBs repair. BLM assembles at stalled replication forks $[4,6]$, which supports the notion 
A

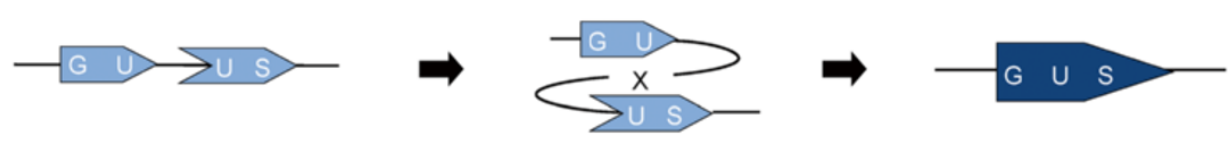

B
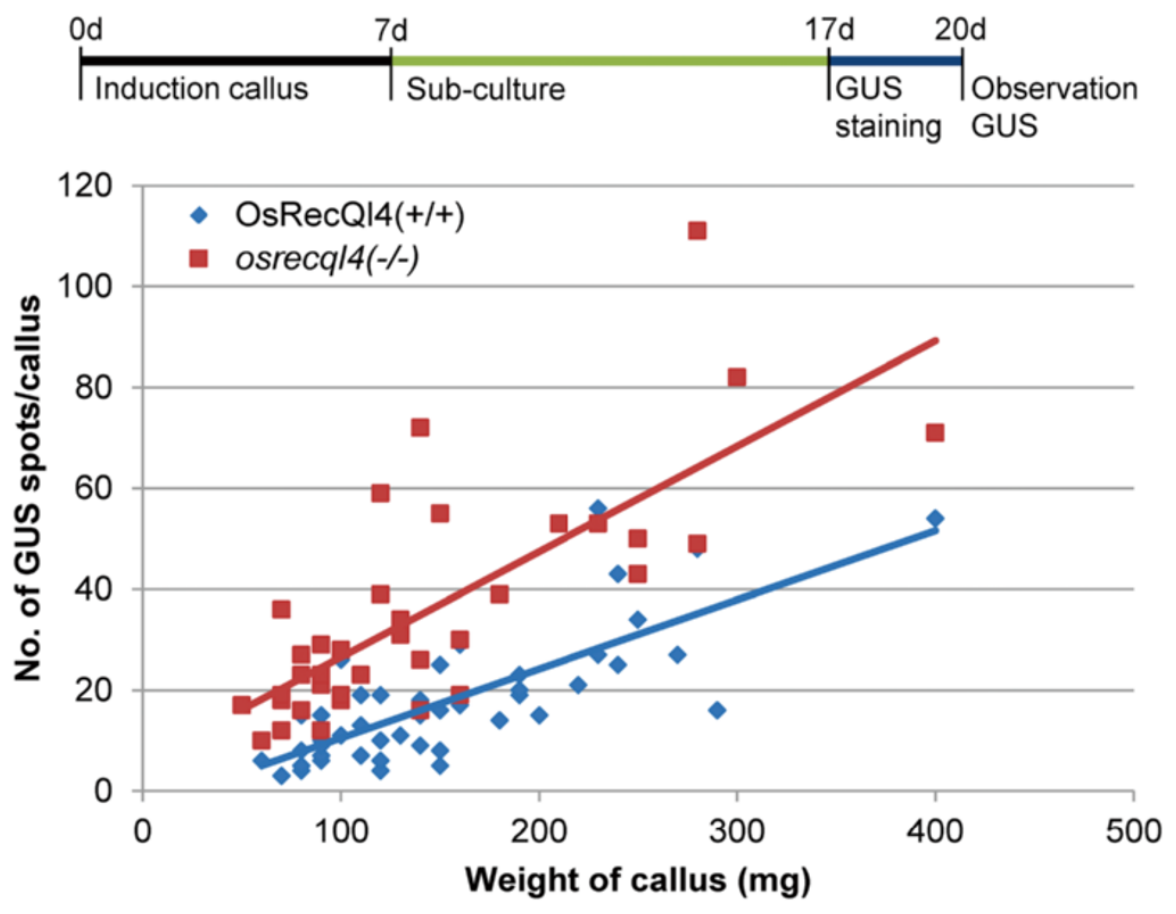

C
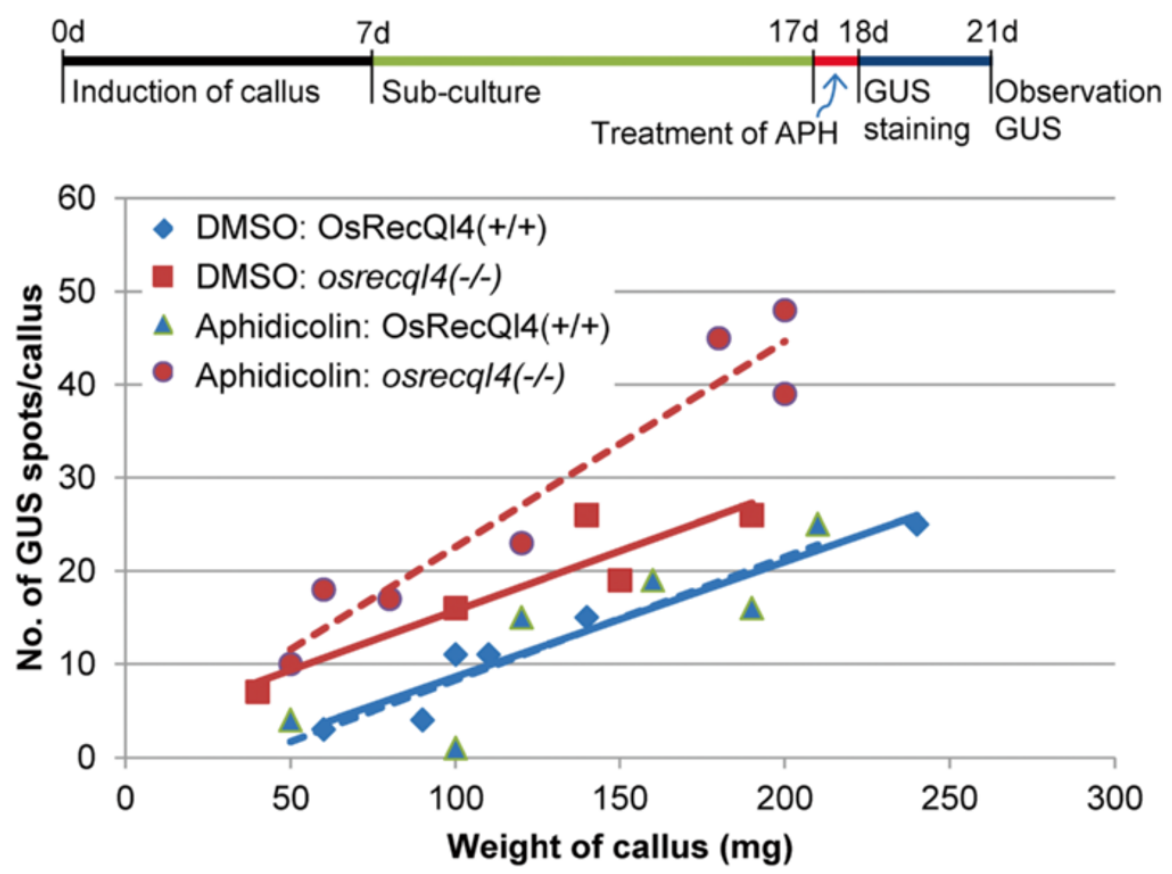

Figure 6 (See legend on next page.) 
(See figure on previous page.)

Figure 6 Enhanced HR in osrecq/4-2 mutants. (A) The GUS recombination reporter (pGU.C.US) used in this experiment is illustrated schematically. Recombination between the two overlapping sequences produces a functional GUS gene. (B) Graph showing the number of GUS blue spots per callus weight as counted under the microscope. HR frequency increased more in the osrecal4-2 mutant than in the WT. GUS histochemical staining was performed on 17-day-old calli. (C)Aphidicolin treatment enhanced HR further in osrecq/4-2 mutants, but HR frequency in WT did not differ from that of the WT without aphidicolin treatment. 17-day-old calli were treated $5 \mathrm{mg} / \mathrm{L}$ aphidicolin for $24 \mathrm{~h}$. The aphidicolintreated calli were then subjected to GUS staining. Blue diamonds OsRecQ|4(+/+); red squares OsRecQ|4(-/-); blue solid line average of OsRecQ|4 $(+/+)$ GUS spots; red solid line average of OsRecQl4(-/-) GUS spots; blue triangles aphidicolin treated OsRecQl4(+/+), red circles aphidicolin treated OsRecQI4(-/-); blue dotted lines average of aphidicolin-treated OsRecQI4(+/+) GUS spots; red dotted line average of aphidicolin-treated OsRecQ14 $(-/-)$ GUS spots.

of DNA replication arrest inducible expression of OsRecQ14 (Additional file 1: Figure S5). These results suggest that OsRecQ14 is required for recovery from DNA replication arrest. The osrecql4-2 mutant failed to recover from DNA replication arrest; this resulted in an increased number of DSBs requiring repair, possibly by HR.

Interestingly, we found that, without aphidicolin treatment, both WT and osrecql4-2 mutants produced a very low level of DSBs (Figure 4) judging from the comet assay. Similarly, DNA damage in osrecql4-2 at the cell division root zone analyzed by TUNEL was almost comparable to that of WT plants. However, the osrecql4-2 mutant showed a greater frequency of HR than did WT plants under normal growth conditions without aphidicolin treatment. This suggested that DSBs induced in the osrecql4-2 mutant could be repaired by DNA repair systems, including HR, under normal growth conditions, since HR can proceed slowly without RecQ helicase [48]. However, excess DSBs induced by aphidicolin treatment could not be repaired by intrinsic DNA repair systems, and un-repaired DSBs were thus detected by comet and TUNEL assay. Furthermore, un-repaired DSBs might induce cell death in osrecql4 mutants upon aphidicolin treatment. Thus, OsRecQl4 might be required to rescue plants from genotoxic stress.

Broadly speaking, RecQ helicases maintain DNA stability. The RecQ helicase BLM promotes not only recovery from DNA replication arrest, but also exonuclease 1-mediated DNA resection during the initial step of DSB repair [49]. OsRecQ14 also plays a role in promoting processing of HR-mediated DSB repair in rice [26]. OsRecQ14 was expressed exclusively in meristems; also, other rice RecQ-like genes were expressed in meristematic tissues [27]. Thus, we anticipate overlapping and distinct roles for the seven RecQ helicase genes in rice.

\section{Conclusions}

The results reported here indicate that OsRecQ14 has an important function in maintaining genome stability in rice during DNA replication. Since BLM and SGS1 exert their function of recovering arrested replication forks by their helicase activity, OsRecQ14 might have the same activity in genome maintenance in rice. In particular, enhanced DSBs and cell death were observed upon DNA replication arrest in the cell division zone of osrecql4 mutants. From this, we conclude that OsRecQ14 is required for recovery from DNA replication arrest in the rice meristem. RecQ helicases should have partially overlapping roles in maintaining genome stability, and among them might be specialists whose primary role is genome stability.

\section{Methods}

\section{Phylogenetic analysis}

We searched the rice full-length cDNA databases, NCBI [http://www.ncbi.nlm.nih.gov/] and GRAMENE [http:// www.gramene.org/], using the programs BioEdit (version 7.0.5.3) and MEGA (version 3.1) to identify rice homologues of RecQ. Seven RecQ homologues are found in rice. Phylogenetic analysis was performed based on the amino acid sequence alignment generated by the built-in CLUSTALW program.

\section{Synthesis of OsRecQ14 cDNA and isolation of osrecq/4 mutant lines}

Total RNA was isolated from 7-day-old seedlings using an RNeasy Plant Mini kit (Qiagen) according to the protocol supplied by the manufacturer. Reverse transcription was performed using ReverTra Ace (Toyobo, Japan) according to the instructions provided by the manufacturer, with total RNA from rice shoot base. Polymerase chain reaction (PCR) and rapid amplification of cDNA ends (RACE) were performed using high fidelity thermostable DNA polymerase, KOD-Plus (Toyobo, Japan). 5'- and 3'-RACE were performed using a GeneRacer kit (Invitrogen, Carlsbad, CA) with gene-specific primers designed according to the genomic DNA sequence. The gene specific primers used for amplification of the coding region of OsRecQl4 were RecQl4_Start1 (GCCATGATA AAGCCAAGGGTCAACT), and primers for amplification of full length cDNA were RecQ14_End1 (ACCCTAGGCTA TTCTGGCGGACTG), RecQ14-5'Race (GATCCGACCAG TTGACCCTTGGCTTT) and RecQ14-3'Race (ACGCGCT GCAAAGACACGTACAAGG). PCR products were cloned into pCR2.1 TOPO vector (Invitrogen, San Diego, CA) for DNA sequencing.

Seeds of the T-DNA tagged line osrecql4-1 and of the Tos17 insertion line osrecql4-2 were obtained from 
POSTECH (line No. 3A-03503) [50] and NIAS collections (line No. NC2763), respectively [51]. Seeds derived from heterozygous plants were cultivated in soil, and those for the T-DNA and Tos17 insertion lines were propagated further in the greenhouse.

The sites of integration of T-DNA and Tos17 were determined by PCR using the following primers: for T-DNA insertion, combinations specific for the left or right border of the respective T-DNA: pGA2715-L1.5 (GGCCAGTGA ATTCACTAGTGATTGC), 3A-03503-498 F (CAATCCA TCCTCGAAAGGCAAT), 3A-03503-498R (ATTCGCGA GGCCATCTCTCT) and for Tos17 insertion: Tos17_3880 (AGTCGCTGATTTCTTCACCAAGG), NC2763-F (TGC CTTGTACGTGTCTTTGC), NC2763-R (AGCTTTGCA ATGCCTTAGGA) and genomic sequences within the respective gene. PCR products were purified and sequenced (Figure 1A).

\section{Northern blot analysis}

Total RNA was prepared from the shoot tips of 10-dayold seedlings using an RNeasy Plant Mini Kit (Qiagen, Valencia, CA). Total RNA (20 $\mu \mathrm{g})$ was loaded and separated on a $1 \%$ agarose gel, and transferred onto a positively charged nylon membrane (Roche, Mannheim, Germany). Probe A (OsRecQ4-proN_F, AACACAAAGGCCTAATC AGGAAGCA; OsRecQ4-proN_R, ATTCCTGGTGTAA ATCGGTGATTGG) or B (RecQ14_1F, GTCGAAAAAG ATGTGACCAACATTGCTAG; RecQ14_1R, TCCCGTCC ACTTGACTCTGTTGATTAG) (Figure 1A) was prepared using a PCR DIG probe synthesis kit (Roche), and hybridization was performed according to the DIG Application Manual (Roche). Hybridization was performed at $50^{\circ} \mathrm{C}$, and washing was performed under high-stringency conditions at $50^{\circ} \mathrm{C}$

\section{Treatment with DNA damaging agents}

To study the effect of DNA damaging agents on rice, We prepared a $10 \mathrm{mg} / \mathrm{ml}$ stock of cisplatin (CDDP, cisdiamminedichloroplatinum [II]; Wako Pure Chemical Industries, Tokyo, Japan), a $10 \mathrm{mg} / \mathrm{ml}$ stock of bleomycin (BLE; Wako Pure Chemical Industries), a $10 \mathrm{mg} / \mathrm{ml}$ stock of aphidicolin (APH; Wako Pure Chemical Industries), a $10 \mathrm{mg} / \mathrm{ml}$ stock of nocodazole (NOC; Wako Pure Chemical Industries), and a $1 \mathrm{mM}$ stock of camptothecin (CPT; Wako Pure Chemical Industries).

To analyze the effect of DNA damaging agents on root elongation, sterilized seeds were sown on MS solid medium containing DNA-damaging agents and grown in a growth chamber at $30^{\circ} \mathrm{C}$ under continuous light. Five days after sowing, root length was measured using Image J software (version 1.43).

To prepare plant materials for PI staining and TUNEL assay, we transferred 5-day-old rice plants grown on MS solid medium to MS liquid medium containing different concentrations of DNA-damaging agents and then incubated for a further 24 hours in the growth chamber. For comet assay, 4-week-old calli grown on N6D solid medium were transferred to N6D liquid medium containing DNA damaging agents and then incubated for a further 1 hour at RT.

\section{PI staining of root tip}

Root tips, which were cut approximately $10 \mathrm{~mm}$ from the root tip, were stained with $5 \mathrm{mg} / \mathrm{L}$ PI (dissolved in sterile water) for $20 \mathrm{~min}$ on a slide glass.

\section{TUNEL (terminal deoxynucleotidyl transferase-mediated dUTP nick and labeling) analysis}

Roots were fixed overnight with $4 \%(\mathrm{v} / \mathrm{v})$ paraformaldehyde in PBS ( $\mathrm{pH} 7.4)$ at $4^{\circ} \mathrm{C}$. Next, fixed roots were dehydrated in a series of tert-butanol and embedded in paraplast for sectioning. The samples were rehydrated in a graded ethanol series to water to remove paraformaldehyde, before being treated with proteinase $\mathrm{K}$ $(20 \mu \mathrm{g} / \mathrm{ml}$ proteinase $\mathrm{K}$ in $10 \mathrm{mM}$ Tris- $\mathrm{HCl}, \mathrm{pH} 7.5)$ at $37^{\circ} \mathrm{C}$ for $30 \mathrm{~min}$, and then washed three times with PBS. TUNEL reaction was performed on a slide glass using the In Situ cell death detection kit with fluorescein (Roche) according to the manufacturer's instructions.

\section{Comet assay}

Microscopic slides were precoated with a layer of $1 \%$ normal melting point agarose and thoroughly dried. Calli were chopped in $200 \mu \mathrm{l}$ PBS containing $50 \mathrm{mM}$ EDTA with razor blade. $30 \mu \mathrm{l}$ of the resulting suspension of nuclei was mixed with an equal volume of liquid $1 \%$ lowmelting-point agarose at $42^{\circ} \mathrm{C}$ and spread on microscope slides pre-coated with $1 \%$ normal-melting-point agarose. After solidification of the agarose containing nuclei, the nuclei were subjected to lysis in a high salt solution (2.5 M NaCl, 10 mM Tris-HCl pH 7.5, 100 mM EDTA) for $20 \mathrm{~min}$ at room temperature. Equilibration for $3 \times$ $5 \mathrm{~min}$ in $1 \times \mathrm{TBE}$ on ice was followed by electrophoresis at room temperature in $1 \times$ TBE buffer for $6 \mathrm{~min}$. To clear gels of the starch grains that are present in the nuclear suspension, the slides were kept for $10 \mathrm{~min}$ in $1 \%$ Triton prior to dehydration for $2 \times 5 \mathrm{~min}$ in $70 \%$ ethanol and $96 \%$ ethanol and air drying. Dry agarose gels were stained with a 1:10,000 diluted SYBR green. After staining, the slides were captured under a fluorescent microscope using Leica DM5000 microscope software and DNA damage was quantitated using CometScore ${ }^{\mathrm{mt}}$ software.

\section{Microscopy}

Images of PI-stained root tips were captured by a Keyence (BZ-9000) microscope with Z-stack function. The Z-stack function moves the focal point of an objective lens through the Z-axis, captures several images, extracts only focused 
points from the images, and synthesizes the points into an omni-focal image (http://www.keyence.com/products/ microscope/fluorescence/bz8000/bz8000_features_3.php). A Leica DM5000 microscope was also used for imagecapture.

\section{Rice transformation}

Agrobacterium-mediated transformation of rice (O. sativa L. cv. Nipponbare) was performed as described previously $[52,53]$. Hulled seeds were sterilized and germinated on N6D medium for 1 week. After co-cultivation of Agrobacterium carrying the pGU.C.US vector, infected calli were washed with sterilized water seven times and with $12.5 \mathrm{mg} / \mathrm{L}$ meropenem three times. The calli were transferred to N6D medium containing suitable antibiotics for selecting transformed cells. Calli that grew vigorously on the selection medium were transferred to regeneration medium containing antibiotics. The regenerated plants were further grown on hormone-free MS medium, and then planted in soil.

\section{Histochemical GUS staining}

Histochemical staining of plant materials was performed as described by Jefferson [54]. To facilitate the penetration of staining buffer, plants were cut into segments of approximately $10 \mathrm{~mm}$, and large calli were divided into several pieces. These plant materials were vacuum infiltrated with X-Gluc staining substrate for $3 \times 10 \mathrm{~min}$ and then incubated at $37^{\circ} \mathrm{C}$ for 3 days. Calli were subsequently bleached with $70 \%$ ethanol and the number of GUS blue spots was counted.

\section{Additional file}

Additional file 1: OsRecQI4-replication-SD-r.

\begin{abstract}
Abbreviations
BIR: Break-induced replication; BS: Base of shoot; dHJ: Double-Holliday junction; DSB: DNA double-strand break; DSE: Double-strand end; HR: Homologous recombination; LS: Lower shoot; NB: Nipponbare; NTS: Non-transcribed spacer; PI: Propidium iodide; POL81: DNA polymerase delta 1; R: Root; RAM: Root apical meristem; rDNA: Ribosomal RNA gene; RPA: Replication protein A; SAM: Shoot apical meristem; SCE: Sister chromatid exchange; SDSA: Synthesis-dependent strand annealing; SSA: Single-strand annealing; SSB: Single-strand break; US: Upper shoot.
\end{abstract}

\section{Competing interests}

The authors declare that they have no competing interests.

\section{Authors' contributions}

YIK led the design of the study and carried out part of the experimental work, KA, ME participated in the design of the study, carried out part of the experimental work, and advised on experimental processes; KO participated in the study design, provided constructs of promoters and terminators etc.; AT carried out part of the in situ hybridization analysis; NO, ANY and HS participated in the study design, and carried out part of the experimental work. ST was overall study supervisor, participating in study design, helping write the manuscript and obtained the funding. All authors read and approved the final version.

\section{Acknowledgments}

We thank Dr. Puchta for provision of recombination substrate, H. Rothnie for English editing of the manuscript, and K. Amagai, A. Nagashii and F. Suzuki for general experimental technical support. N. Kishimoto assisted in statistical analysis of PI staining. This research was supported by a scholarship to Y.I. Kwon from the Ministry of Education, Culture, Sports, Science and Technology of Japan (MEXT).

This work was also supported by a PROBRAIN (Program for Promotion of Basic Research Activities for Innovative Biosciences) grant to S. Toki from the Bio-Oriented Technology Research Advancement Institution (BRAIN) of Japan; a grant from the Ministry of Agriculture, Forestry and Fisheries of Japan (Genomics for Agricultural Innovation, GMC-0001); and the Strategic Promotion Program for Basic Nuclear Research (No. 210105, No. 23658012) of the Ministry of Education, Culture, Sports, Science and Technology of Japan.

\section{Author details}

${ }^{1}$ Plant Genome Engineering Research Unit, National Institute of Agrobiological Sciences, 2-1-2 Kannondai, Tsukuba, Ibaraki 305-8602, Japan. ${ }^{2}$ Functional Plant Research Unit, National Institute of Agrobiological Sciences, 2-1-2 Kannondai, Tsukuba, Ibaraki 305-8602, Japan. ${ }^{3}$ Graduate School of Science and Engineering, Saitama University, 255 Shimo-okubo, Sakura, Saitama 338-8570, Japan. ${ }^{4}$ Graduate School of Nanobioscience, Yokohama City University, 22-2, Seto, Kanazawa, Yokohama 236-0027, Japan. ${ }^{5}$ Kihara Institute for Biological Research, Yokohama City University, 641-12 Maioka, Yokohama 244-0813, Japan.

Received: 20 December 2012 Accepted: 3 April 2013

Published: 12 April 2013

\section{References}

1. Bohr VA: Rising from the RecQ-age: the role of human RecQ helicases in genome maintenance. Trends Biochem Sci 2008, 33(12):609-620.

2. Singh DK, Ahn B, Bohr VA: Roles of RECQ helicases in recombination based DNA repair, genomic stability and aging. Biogerontology 2009, 10(3):235-252.

3. Bachrati CZ, Hickson ID: RecQ helicases: guardian angels of the DNA replication fork. Chromosoma 2008, 117(3):219-233.

4. Wu L: Role of the BLM helicase in replication fork management. DNA Repair (Amst) 2007, 6(7):936-944.

5. Singh DK, Ghosh AK: Croteau DL. Bohr VA: RecQ helicases in DNA double strand break repair and telomere maintenance. Mutat Res; 2011.

6. Rezazadeh S: RecQ helicases; at the crossroad of genome replication, repair, and recombination. Mol Biol Rep 2012, 39(4):4527-4543.

7. Wu L, Hickson ID: RecQ helicases and topoisomerases: components of a conserved complex for the regulation of genetic recombination. Cell Mol Life Sci 2001, 58(7):894-901.

8. Hartung F, Puchta H: The RecQ gene family in plants. J Plant Physiol 2006, 163(3):287-296.

9. Rossi ML, Ghosh AK, Bohr VA: Roles of Werner syndrome protein in protection of genome integrity. DNA Repair (Amst) 2010, 9(3):331-344.

10. Ellis NA, Groden J, Ye TZ, Straughen J, Lennon DJ, Ciocci S, Proytcheva M, German J: The Bloom's syndrome gene product is homologous to RecQ helicases. Cell 1995, 83(4):655-666.

11. Gray MD, Shen JC, Kamath-Loeb AS, Blank A, Sopher BL, Martin GM, Oshima J, Loeb LA: The Werner syndrome protein is a DNA helicase. Nat Genet 1997, 17(1):100-103.

12. Kitao S, Shimamoto A, Goto M, Miller RW, Smithson WA, Lindor NM, Furuichi Y: Mutations in RECQL4 cause a subset of cases of Rothmund-Thomson syndrome. Nat Genet 1999, 22(1):82-84.

13. Nimonkar AV, Ozsoy AZ, Genschel J, Modrich P, Kowalczykowski SC: Human exonuclease 1 and BLM helicase interact to resect DNA and initiate DNA repair. Proc Natl Acad Sci U S A 2008, 105(44):16906-16911.

14. Mimitou EP, Symington LS: Sae2, Exo1 and Sgs1 collaborate in DNA double-strand break processing. Nature 2008, 455(7214):770-774

15. McDaniel LD, Schultz RA: Elevated sister chromatid exchange phenotype of Bloom syndrome cells is complemented by human chromosome 15. Proc Natl Acad Sci U S A 1992, 89(17):7968-7972.

16. Brosh RM Jr, Li JL, Kenny MK, Karow JK, Cooper MP, Kureekattil RP, Hickson ID, Bohr VA: Replication protein A physically interacts with the Bloom's syndrome protein and stimulates its helicase activity. J Biol Chem 2000, 275(31):23500-23508. 
17. Wu L, Davies SL, North PS, Goulaouic H, Riou JF, Turley H, Gatter KC, Hickson ID: The Bloom's syndrome gene product interacts with topoisomerase III. J Biol Chem 2000, 275(13):9636-9644.

18. Davies SL, North PS, Dart A, Lakin ND, Hickson ID: Phosphorylation of the Bloom's syndrome helicase and its role in recovery from S-phase arrest. Mol Cell Biol 2004, 24(3):1279-1291.

19. Davies SL, North PS, Hickson ID: Role for BLM in replication-fork restart and suppression of origin firing after replicative stress. Nat Struct Mol Biol 2007, 14(7):677-679

20. Lonn U, Lonn S, Nylen U, Winblad G, German J: An abnormal profile of DNA replication intermediates in Bloom's syndrome. Cancer Res 1990, 50(11):3141-3145.

21. Karow JK, Constantinou A, Li JL, West SC, Hickson ID: The Bloom's syndrome gene product promotes branch migration of holliday junctions. Proc Natl Acad Sci U S A 2000, 97(12):6504-6508.

22. Saintigny $Y$, Delacote F, Vares G, Petitot F, Lambert S, Averbeck D, Lopez BS: Characterization of homologous recombination induced by replication inhibition in mammalian cells. EMBO J 2001, 20(14):3861-3870.

23. Bagherieh-Najjar MB, de Vries OMH, Hille J, Dijkwel PP: Arabidopsis RecQ14A suppresses homologous recombination and modulates DNA damage responses. Plant J 2005, 43(6):789-798.

24. Hartung F, Suer S, Puchta H: Two closely related RecQ helicases have antagonistic roles in homologous recombination and DNA repair in Arabidopsis thaliana. Proc Natl Acad Sci U S A 2007, 104(47):18836-18841.

25. Mannuss A, Dukowic-Schulze S, Suer S, Hartung F, Pacher M, Puchta H: RAD5A, RECQ4A, and MUS81 Have Specific Functions in Homologous Recombination and Define Different Pathways of DNA Repair in Arabidopsis thaliana. Plant Cell 2010, 22(10):3318-3330.

26. Kwon Yl, Abe K, Osakabe K, Endo M, Nishizawa-Yokoi A, Saika H, Shimada H, Toki S: Overexpression of OsRecQ14 and/or OsExo1 Enhances DSB-Induced Homologous Recombination in Rice. Plant Cell Physio/ 2012 53(12):2142-2152.

27. Saotome A, Kimura S, Mori Y, Uchiyama Y, Morohashi K, Sakaguchi K: Characterization of four RecQ homologues from rice (Oryza sativa L. CV. Nipponbare). Biochem Biophys Res Commun 2006, 345(4):1283-1291.

28. Kotogany E, Dudits D, Horvath GV, Ayaydin F: A rapid and robust assay for detection of S-phase cell cycle progression in plant cells and tissues by using ethynyl deoxyuridine. Plant Methods 2010, 6(1):5

29. Endo M, Nakayama S, Umeda-Hara C, Ohtsuki N, Saika H, Umeda M, Toki S: CDKB2 is involved in mitosis and DNA damage response in rice. Plant J 2012, 69(6):967-977.

30. Adachi S, Minamisawa K, Okushima Y, Inagaki S, Yoshiyama K, Kondou Y, Kaminuma E, Kawashima M, Toyoda T, Matsui M, et al: Programmed induction of endoreduplication by DNA double-strand breaks in Arabidopsis. Proc Natl Acad Sci U S A 2011, 108(24):10004-10009.

31. Schwartz S, Kent WJ, Smit A, Zhang Z, Baertsch R, Hardison RC, Haussler D, Miller W: Human-mouse alignments with BLASTZ. Genome Res 2003, 13(1):103-107

32. Sato $Y$, Antonio BA, Namiki N, Takehisa H, Minami H, Kamatsuki K, Sugimoto K, Shimizu Y, Hirochika H, Nagamura Y: RiceXPro: a platform for monitoring gene expression in japonica rice grown under natural field conditions. Nucleic Acids Res 2011, 39(Database issue):1141-1148.

33. Spadari S, Pedrali-Noy G, Falaschi MC, Ciarrocchi G: Control of DNA replication and cell proliferation in eukaryotes by aphidicolin. Toxicol Pathol 1984, 12(2):143-148.

34. Negoescu A, Lorimier P, Labat-Moleur F, Drouet C, Robert C, Guillermet C, Brambilla C, Brambilla E: In situ apoptotic cell labeling by the TUNEL method: improvement and evaluation on cell preparations. J Histochem Cytochem 1996, 44(9):959-968.

35. Wang C, Liu Z: Arabidopsis ribonucleotide reductases are critical for cell cycle progression, DNA damage repair, and plant development. Plant Cell 2006, 18(2):350-365.

36. Shishkova S, Dubrovsky JG: Developmental programmed cell death in primary roots of Sonoran Desert Cactaceae. Am J Bot 2005, 92(9):1590-1594

37. Siebert R, Puchta H: Efficient repair of genomic double-strand breaks by homologous recombination between directly repeated sequences in the plant genome. Plant Cell 2002, 14(5):1121-1131.

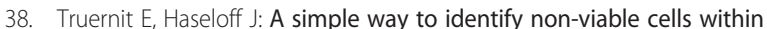
living plant tissue using confocal microscopy. Plant Methods 2008, 4(1):15.
39. Urawa H, Hidaka M, Ishiguro S, Okada K, Horiuchi T: Enhanced homologous recombination caused by the non-transcribed spacer of the rDNA in Arabidopsis. Mol Genet Genomics 2001, 266(4):546-555

40. Keil RL, Roeder GS: Cis-acting, recombination-stimulating activity in a fragment of the ribosomal DNA of S. cerevisiae. Cell 1984, 39(2 Pt 1):377-386

41. McGlynn P, Lloyd RG: Recombinational repair and restart of damaged replication forks. Nat Rev Mol Cell Biol 2002, 3(11):859-870.

42. Schuermann D, Fritsch O, Lucht JM, Hohn B: Replication stress leads to genome instabilities in Arabidopsis DNA polymerase delta mutants. Emphasis Type="ltalic" $>$ Plant Cell 2009, 21(9):2700-2714.

43. Heyer WD, Ehmsen KT, Liu J: Regulation of homologous recombination in eukaryotes. Annu Rev Genet 2010, 44:113-139.

44. Roth N, Klimesch J, Dukowic-Schulze S, Pacher M, Mannuss A, Puchta H: The requirement for recombination factors differs considerably between different pathways of homologous double-strand break repair in somatic plant cells. Plant J 2012, 72(5):781-790.

45. Shrivastav M, De Haro LP, Nickoloff JA: Regulation of DNA double-strand break repair pathway choice. Cell Res 2008, 18(1):134-147.

46. Allen C, Ashley AK, Hromas R, Nickoloff JA: More forks on the road to replication stress recovery. $J \mathrm{Mol}$ Cell Biol 2011, 3(1):4-12.

47. Chester N, Kuo F, Kozak C, O'Hara CD, Leder P: Stage-specific apoptosis, developmental delay, and embryonic lethality in mice homozygous for a targeted disruption in the murine Bloom's syndrome gene. Genes Dev 1998, 12(21):3382-3393.

48. Zhu Z, Chung WH, Shim EY, Lee SE, Ira G: Sgs1 helicase and two nucleases Dna2 and Exo1 resect DNA double-strand break ends. Cell 2008, 134(6):981-994.

49. Bernstein KA, Gangloff S, Rothstein R: The RecQ DNA Helicases in DNA Repair. Annu Rev Genet 2010, 44:393-417.

50. Jeong DH, An S, Kang HG, Moon S, Han JJ, Park S, Lee HS, An K, An G: T-DNA insertional mutagenesis for activation tagging in rice. Plant Physiol 2002, 130(4):1636-1644.

51. Miyao A, Tanaka K, Murata K, Sawaki H, Takeda S, Abe K, Shinozuka Y, Onosato K, Hirochika $\mathrm{H}$ : Target site specificity of the Tos 17 retrotransposon shows a preference for insertion within genes and against insertion in retrotransposon-rich regions of the genome. Plant Cell 2003, 15(8):1771-1780.

52. Toki S: Rapid and efficient Agrobacterium-mediated transformation in rice. Plant Mol Biol Rep 1997, 15(1):16-21.

53. Toki S, Hara N, Ono K, Onodera H, Tagiri A, Oka S, Tanaka H: Early infection of scutellum tissue with Agrobacterium allows high-speed transformation of rice. Plant J 2006, 47(6):969-976

54. Jefferson RA, Kavanagh TA, Bevan MW: GUS fusions: beta-glucuronidase as a sensitive and versatile gene fusion marker in higher plants. EMBO J 1987, 6(13):3901-3907.

doi:10.1186/1471-2229-13-62

Cite this article as: Kwon et al:: DNA replication arrest leads to enhanced homologous recombination and cell death in meristems of rice OsRecQ14 mutants. BMC Plant Biology 2013 13:62.

\section{Submit your next manuscript to BioMed Central and take full advantage of:}

- Convenient online submission

- Thorough peer review

- No space constraints or color figure charges

- Immediate publication on acceptance

- Inclusion in PubMed, CAS, Scopus and Google Scholar

- Research which is freely available for redistribution 